\title{
Nutrition assessment methods for the older Irish adult in the clinical and community settings
}

\author{
Ruth P. Charles \\ Department of Nutrition and Dietetics, Adelaide and Meath Hospital Dublin incorporating the \\ National Children's Hospital, Tallaght, Dublin 24, Republic of Ireland
}

What is nutritional assessment? As referred to in the present context, it is a process where several assessments are combined to enable the evaluation of an individual's nutritional status. It usually comprises aspects of clinical, anthropometric, biochemical, functional or other data. Its overall aim is to provide useful variables to define the nutritional status of the older Irish adult in community and clinical settings. For the purpose of defining the older Irish adult a cut-off age of 65 years is used.

The principal reason for continued focus on nutritional status in the older adult is due to the widespread occurrence of undernutrition in this age-group (Bianchetti et al. 1990; Mowé \& Bøhmer, 1991; Keller, 1993). Undernutrition is a condition which results when an individual is below normal or ideal weight, with an underconsumption of most or all nutrients, and has an inadequate nutritional status (Charles et al. 1998).

Undernutrition in this age-group is associated with higher morbidity and mortality (Sullivan et al. 1990), delayed functional recovery (Dàvalos et al. 1996), impaired immunocompetence (Chandra, 1983), delayed wound healing (Closs, 1993), organ system dysfunction (Doekel et al. 1976) and more frequent hospitalization (Williams \& Fitton, 1988; Sullivan, 1992). Nutritional assessment has several prognostic implications, and nutritional variables are now considered a cardinal component of multidisciplinary assessment in the acute care setting (Incalzi et al. 1996). Nutritional assessment can take two forms: a screen which identifies those 'at risk' individuals (Charles et al. 1998), or a detailed assessment of risk degree and severity using a score system which when repeated at subsequent intervals can map the risk progression and even give an indication of the success of any intervention (Guigoz et al. 1994).

Nutrition assessment can also be used to initiate and adapt nutrition care plans for individuals or groups.

\section{Difficulties of nutritional assessment for this age-group}

Currently no 'gold standard' assessment exists which, to date, is practical, efficient, valid and reliable enough to warrant routine use in the Irish clinical or community geriatric assessment settings. Interpretation of information gained from any assessment is of paramount importance to the usefulness of results.
The Mini Nutritional Assessment (Guigoz et al. 1994) is available and fully validated, but necessitates estimation of height and requires some degree of skill in the use of anthropometric techniques and basic calculations. It is a useful tool for assessing the degree of nutritional compromise over time, but is not as useful for routine use in the Irish clinical or community settings due to the length of time and the skills required to complete it.

\section{Hallmarks of good nutritional assessment for the older adult}

For nutritional assessment to be of maximum use and relevance it should incorporate some of, or all, the following aspects:

it should be specific for over 65 year olds;

it should not take longer than 5-10 min to perform and record;

it should be reliable and accurate, i.e. validated;

it should not require extensive complicated equipment;

it should be non-invasive;

it should give a useful and/or meaningful result;

it should be easily repeatable over time.

\section{Elements of nutritional assessment Clinical evaluation}

This has been described as the cornerstone of nutritional assessment for this age-group (Incalzi et al. 1996). It has been defined as the subjective assessment of nutritional status based on historical data and physical findings (Detsky et al. 1987). Historical data can indicate where previous medical or surgical conditions are likely to have an effect on current nutritional status, e.g. stroke, neurological disorder, bowel resection. McLaren (1988) has described well-known links between comprised nutrition and physical findings in relation to skin, hair and nails. Although clinical signs of deficiency may take some time to appear, it is worth looking at these tissues as they are easily examined.

Current diagnosis can also have serious consequences on nutritional status, e.g. infective events, congestive cardiac failure, anorexia. 
Palpation of relevant muscle and fat stores can provide a non-invasive estimate of muscle and fat status, and can perhaps provide some indication of sarcopenia (lower than normal muscle mass or greater than normal rate of loss of muscle tissue with age). Muscle and fat wastage are important predictors of mortality in critical illness and outcome in malignancy and acute illness (Chumlea et al. 1996).

\section{Functional evaluation}

This can be an important predictor of muscle mass, and can be assessed non-invasively by measuring grip strength (Kwok \& Whitelaw, 1991), $10 \mathrm{~m}$ walking time, time taken to rise from a chair, or ability to stand on one leg. Good predictors of independence of function generally correlate well with lean body mass and muscle mass. Muscle strength is one of the best predictors of independence and mobility, and strength is directly determined by the amount of muscle mass (Worid Health Organization, 1995).

\section{Anthropometry}

This is the measure of body size, weight and proportion. It can infer information about amounts of skeletal mass, muscle mass and fatness (Chumlea et al. 1996). Anthropometric measurements are good predictors of ill health, functional impairment and mortality. Measurements obtained are meaningful as long as: body systems are in homeostasis; the measurer is trained in anthropometric techniques; equipment required is available and standardized; the body sites used are standardized. The values obtained in the older adult will be affected by reduction in stature (body height declines by $10-20 \mathrm{~mm}$ per decade after the age of 55 years), changes in the amount and distribution of body fat, and altered tissue elasticity and compressibility (Chumlea et al. 1996).

Little information regarding the use and suitability of anthropometry in older adults was available until Chumlea et al. (1987) standardized methodology, recommended equipment and established American reference values. Seven measurement sites were revealed as useful indicators of nutritional status (stature, weight, knee height, calf circumference, mid-arm circumference, triceps skinfold thickness, subscapular skinfold thickness). Four computed measurements provide additional useful data (stature from knee height, computed weight, weight divided by stature squared, mid-arm muscle area).

The reference values obtained are not suitable for our Irish elderly, as it is recommended that for reference values to be of use the groups should be genetically and ethnically similar. Even though reference data are available from other Celtic populations (Burr \& Phillips, 1984; Lehmann et al. 1991) and our European cousins (De Groot et al. 1992), the World Health Organization (1995) recommend the collection of data to describe local levels and patterns rather than universal reference data. The World Health Organization (1995) have recently reported on the use and interpretation of anthropometry in over 65 year olds. It has highlighted that in individuals who are not in ideal health, anthropometry has limitations both in the application of methods and in interpretation of results. It also recommends that clinicians recognize the limitations of using anthropometry in individuals. Trans- lating this to the Irish setting, detailed anthropometry may be best reserved for the research setting, using the techniques recommended as a guideline. For individuals, specific measurements may be useful longitudinally where the individual acts as their own reference. More detailed Irish reference data are eagerly awaited.

\section{Biochemical assays}

Many assays exist for the measurement of tissue, serum and plasma proteins, vitamins and minerals. They vary in their availability, cost and relevance to actual nutritional status. Interpretation of results can cause confusion, as a low serum value may not always indicate nutritional compromise or clinical deficiency.

Under ideal conditions, transferrin, prealbumin and retinol-binding protein are more sensitive indicators of nutritional status than albumin. However, serum albumin is related to skeletal muscle mass in elderly men and women (Baumgartner et al. 1996). The importance of adequate muscle stores has already been discussed. It is also suggested that the increased risk of disability associated with low serum albumin concentrations may reflect a relationship with sarcopenia. This relationship is independent of other factors that may affect muscle mass and serum albumin concentration (Baumgartner et al. 1996). Serum albumin is also thought to be the simplest and best predictor of mortality, and can provide early identification of those elderly at increased risk of death (Agarwal et al. 1988).

\section{What should nutritional assessment incorporate to be effective and useful for the older Irish adult in clinical and community settings?}

I suggest that nine important elements should be addressed:

1. current weight;

2. weight history;

3. clinical assessment of the individual;

4. grip strength evaluation;

5. serum albumin;

6. calf circumference;

7. recording assessment findings;

8. taking action on assessment findings;

9. repeating assessment on a defined basis.

\section{Current weight and weight history}

Weight is a relatively easy measurement to obtain given the correct equipment. When assessing weight it is important to use patient-specific equipment which is regularly calibrated. Weight is clinically important as an independent predictor of increased mortality. Lehmann et al. (1991) have described a range of desirable weights for the older adult. Medical screening is recommended for those individuals who are below the minimum desired weight-for-health (below the 10th centile) described in Table 1 .

It is essential to compile a trend of weight over time to establish a weight history. A 4-5\% loss of usual weight over a 1-year period is clinically significant (Wallace et al. 1995). Weight loss of this magnitude is associated with loss of 
Table 1. Weight centiles (kg) for over 65 year olds (Adapted from Lehmann et al. 1991)

\begin{tabular}{lccccc}
\hline & \multicolumn{2}{c}{$65-74$ years } & & \multicolumn{2}{c}{$75+$ years } \\
\cline { 2 - 3 } \cline { 5 - 6 } Percentile & Male & Female & & Male & Female \\
\hline 10 & 57 & 50 & & 53 & 45 \\
20 & 62 & 54 & & 57 & 49 \\
30 & 65 & 57 & & 62 & 52 \\
40 & 68 & 60 & & 66 & 56 \\
50 & 71 & 63 & & 69 & 59 \\
60 & 75 & 66 & & 72 & 62 \\
70 & 79 & 69 & & 76 & 66 \\
80 & 84 & 74 & & 78 & 69 \\
90 & 89 & 83 & & 84 & 74 \\
\hline
\end{tabular}

skeletal muscle, decreased bone mass, restricted reserves and decreased compensatory abilities (Wallace et al. 1995). For an individual who has been underweight for the majority of adulthood the prognosis is not as poor as that for an individual whose weight has recently started to decline and is now below the 10th centile. It is advisable to assess weight every 3 months. It is preferable to compare current weight with that obtained 3, 6,9 and 12 months previously. For those who are actively losing weight, regular weekly weight checks are indicated.

\section{Clinical assessment}

Where an individual is hospitalized or visiting the general practitioner, clinical assessment is usually easily performed, and information is readily accessible. Current diagnosis or presenting complaint can give an indication of the likelihood of the development of comprised nutrition. Respiratory or urinary tract infections, stroke, cancer and neurological conditions are just a few that predispose to poor nutritional status and undernutrition in the older adult. Medical (e.g. diabetes, hyperlipidaemia) and surgical (e.g. bowel resection) history can indicate where nutritional status has been an issue in the past. Palpation of the deltoids, quadriceps and mid-axillary line of the lower ribs can provide a good subjective assessment of muscle and fat stores which can further enhance the findings of the complete nutritional assessment (Detsky et al. 1987).

\section{Grip strength}

Its assessment is non-invasive, easy to perform and does not require specialized equipment, although a hand dynamometer can be used if available. When assessed in conjunction with palpation, as described previously, it can indicate where muscle stores are compromised and functional abilities are impaired. This has serious consequences for those who require active rehabilitation.

\section{Serum albumin}

This is an assay that is widely available and easily performed. Its cost is reasonable and, provided that the results obtained are interpreted with the patient's overall medical and surgical status in mind, it is a very useful variable to incorporate into nutritional assessment.

\section{Calf circumference}

This is the anthropometric measurement most recommended as a sensitive measure of the loss of total body mass (Chumlea et al. 1996). The technique for its measurement is easily learned; a measuring tape is all that is required. Repeated measurements over time can be used to recognize a trend.

\section{Recording assessment findings}

This may sound so simple as to not warrant mention, but the key to successful assessment can lie in the manner in which data are recorded and presented. Careful note of all assessments, evaluations and measurements should be made along with the results obtained. When the nutritional assessment is completed in its entirety, a decision should be made as to the significance of the results obtained.

\section{Take action!}

It is now appropriate and timely that the significance of the patient's entire nutritional assessment is carefully considered and an appropriate course of action is taken. This should also be clearly documented.

\section{Repeated assessments}

It will be necessary to define the frequency at which repeated assessments are required. This will vary greatly depending on the individual. For those whose assessment shows clear and definite risk of undernutrition, weekly reassessments are usually indicated to ensure that appropriate action has been taken to halt further nutritional deterioration. It is unlikely that there are individuals over the age of 65 years who are not at risk of nutritional compromise. There are those who are at very low risk and may remain so for some time. The only real method of detecting nutritional decline is to assess this low-risk group on a 6-monthly or yearly basis, such that any nutritional compromise can be detected as soon as it occurs. For those who lie between the two extremes reassessment deadlines are difficult to set, but should be attempted realistically and with the individual in mind.

Repeated assessments should be undertaken in a format identical to that of the original, and preferably by the same observer. The patient can be used as his or her own control.

Ideally a thorough nutritional assessment should comprise all nine elements previously described. Until such a time that a gold standard assessment specific to the Irish older adult's needs has been developed and validated, nutritional assessment should never be based only on one or two elements, as this can lead to gross inaccuracies. The more elements available the better the assessment.

The schema previously described can easily be learned by primary care specialists and non-nutrition subspecialists, but it is always wise to seek the advice and guidance of a qualified nutritionist when formulating assessment techniques. With the high prevalence of undernutrition and its associated conditions, assessment, identification and management of undernutrition should be a component of all practices associated with the care of the older Irish adult. 


\section{References}

Agarwal N, Acevedo F, Leighton LS, Cayten CG \& Pitchumoni CS (1988) Predictive ability of various nutritional variables for mortality in elderly people. American Journal of Clinical Nutrition 48, 1173-1178.

Baumgartner RN, Koehler KM, Romero L \& Garry PJ (1996) Serum albumin is associated with skeletal muscle in eiderly men and women. American Joumal of Clinical Nutrition 64, $552-558$

Bianchetti A, Rozzini R \& Carabellese C (1990) Nutritional intake, socio-economic conditions and health status in a large elderly population. Journal of the American Geriatrics Society 38, 521-526.

Burr ML \& Phillips KM (1984) Anthropometric norms in the elderly. British Journal of Nutrition 51, 165-169.

Chandra RK (1983) Nutrition, immunity and infection: present knowledge and future directions. Lancet iii, 688-691.

Charles RP, Mulligan S \& O'Neill D (1998) The identification and assessment of undernutrition in patients admitted to the Age Related Health Care unit of an acute Dublin teaching hospital. Irish Journal of Medical Science (In the Press).

Chumlea WC, Gou SS, Vellas B \& Guigoz Y (1996) Assessing body composition and sarcopenia with anthropometry. In Proceedings from Cerin Symposium: Nutrition \& Personnes Âgées, Au-dela Des Apports Recommandés (Nutrition and the Elderly According to Recommended Guidelines), pp. 161-169. Paris: Cerin.

Chumlea WC, Roche AF \& Mukherjee D (1987) Nutritional Assessment of the Elderly Through Anthropometry. Columbus, $\mathrm{OH}$ : Ross Laboratories.

Closs SJ (1993) Malnutrition: the key to pressure sores? Nursing Standard 8, 32-36.

Dàvalos A, Ricart W \& Gonzalez-Huiz F (1996) Effect of malnutrition after acute stroke on clinical outcome. Stroke 27, $1028-1032$

De Groot CPGM, Hautvast JGAJ \& van Stavern WA (1992) Nutrition and health of elderly people in Europe: the EURONUT-SENECA study. Nutrition Reviews 50, 185-194.

Detsky AS, McLaughlin JR, Baker JP, Johnson N, Whittaker S, Mendelson RA \& Jeejeebhoy KN (1987) What is subjective global assessment of nutritional status? Journal of Parenteral and Enteral Nutrition 11, 8-13.

Doekel RC, Zwillich CW \& Scoggin CH (1976) Clinical semistarvation: depression of hypoxic ventilatory response. New England Journal of Medicine 295, 358-361.
Guigoz Y, Vellas B \& Garry PJ (1994) Mini Nutritional Assessment: a practical assessment tool for grading the nutritional state of elderly patients. Facts and Research in Gerontology 4, Suppl. $2,15-59$.

Incalzi RA, Landi F, Cipriani L, Bruno E, Pagano F, Gemma A, Capparella O \& Carbonin P (1996) Nutritional assessment: a primary component of multidimensional geriatric assessment in the acute care setting. Journal of the American Geriatrics Society 44, 166-174.

Keller HH (1993) Malnutrition in institutionalized elderly: how and why? Journal of the American Geriatrics Society 41, 1212-1218.

Kwok T \& Whitelaw MN (1991) The use of armspan in nutritional assessment of the elderly. Journal of the American Geriatrics Society 39, 492-496.

Lehmann AB, Bassey EJ, Morgan K \& Dallosso HM (1991) Normal values for weight, skeletal size and body mass indices in 890 men and women aged over 65 years. Clinical Nutrition 10, $18-22$.

McLaren DS (1988) Clinical manifestations of nutritional disorders. In Modern Nutrition in Health and Disease, 7th ed., pp. 733-745 [ME Shils and VR Young, editors]. Philadelphia, PA: Lea \& Febiger.

Mowé M \& Bøhmer T (1991) The prevalence of undiagnosed protein-calorie undernutrition in a population of hospitalized elderly patients. Journal of the American Geriatrics Society 39. 1089-1092.

Sullivan DH (1992) Risk factors for early hospital readmission in a select population of Geriatric rehabilitation patients: the significance of nutritional status. Journal of the American Geriatrics Society 40, 792-798.

Sullivan DH, Patch GA \& Walls RC (1990) Impact of nutrition status on morbidity and mortality in a select population of Geriatric rehabilitation patients. American Journal of Clinical Nutrition 51, 749-758.

Wallace JI, Schwartz RS, LaCroix AZ, Uhlmann RF \& Pearlman RA (1995) Involuntary weight loss in older outpatients: incidence and clinical significance. Journal of the American Geriatrics Society 43, 329-337.

Williams EI \& Fitton F (1988) Factors affecting early unplanned readmission of elderly patients to hospital. British Medical Journal 297, 784-787.

World Health Organization (1995) Physical Status: The Use and Interpretation of Anthropometry. Report of a WHO Expert Committee. World Health Organization Technical Report Series no. 854. Geneva: WHO. 